\title{
UNA APROXIMACIÓN A LA METODOLOGÍA DE INVESTIGACIÓN DE LOS CRÍMENES DE LESA HUMANIDAD EN LAS DICTADURAS DEL CONO SUR.
}

\author{
LA EXPERIENCIA DEL EQUIPO DE \\ INVESTIGACIÓN HISTÓRICA (EIH) URUGUAY.
}

\author{
An approach to the methodology of investigation of \\ crimes against humanity in the dictatorships of the \\ southern cone.
}

The experience of the Historical Research Team (HRT) Uruguay.

\author{
Magdalena Figueredo Corradi* \\ Fabiana Larrobla Caraballo**
}

\begin{abstract}
RESUMEN
Las distintas investigaciones sobre las denuncias de violación a los derechos humanos sucedidas en las últimas dictaduras del siglo XX han significado una suma de nuevos desafíos y debates al interior de diversas disciplinas sociales y jurídicas, tanto en lo que tiene que ver con las dificultades de su abordaje como en la discusión sobre el rol de los y las investigadoras en la configuración de un nuevo campo. El presente artículo trata sobre el proceso de construcción de un abordaje metodológico desde las ciencias sociales y humanas enmarcada en el enfoque transdisciplinar, que se origina a partir del trabajo realizado durante más de quince años en la investigación sobre los crímenes
\end{abstract}

\footnotetext{
* Equipo de Investigación Histórica. Secretaría de Derechos Humanos para el Pasado Reciente. Presidencia de la República. E-mail: magdalenafigueredo@gmail.com.

** Coordinadora del Equipo de Investigación Histórica. Secretaría de Derechos Humanos para el Pasado Reciente. Presidencia de la República. E-mail: fabianalarro@gmail.com
} 
cometidos por el estado uruguayo dentro de su territorio y en el marco del Plan Cóndor. Esta propuesta metodológica pretende brindar las herramientas necesarias para poder llevar adelante investigaciones de corte académico que tengan entre sus propósitos contribuir a los procesos de verdad, justicia y reparación en relación con las graves violaciones a los derechos humanos cometidas en las últimas dictaduras del siglo XX en el cono sur. El contenido del artículo forma parte de un proyecto más amplio destinado a generar un campo de estudio en la materia que permita seguir avanzando en la construcción de estas herramientas.

Palabras claves: Dictadura en Uruguay; Investigaciones sobre Pasado Reciente; Transdisciplina.

\begin{abstract}
The different investigations on the denunciations of violation of the human rights happened in the last dictatorships of the twentieth century have meant a sum of new challenges and debates inside diverse social and legal disciplines, so much in which it has to do with the difficulties of its approach as in the discussion about the role of researchers in the configuration of a new field. This article is about the process of building a methodological approach from the social and human sciences framed in the transdisciplinary approach, which originates from the work carried out for more than fifteen years in the investigation of crimes committed by the Uruguayan state within of its territory and within the framework of the Condor Plan. This methodological proposal aims to provide the necessary tools to carry out academic investigations that have among their purposes to contribute to the processes of truth, justice and reparation in relation to the serious human rights violations committed in the last dictatorships of the twentieth century in the southern cone. The content of the article is part of a larger project aimed at generating a field of study that allows further progress in the construction of these tools.
\end{abstract}

Keywords: Dictatorship in Uruguay; Recent Past Research; Transdiscipline.

Las distintas investigaciones sobre las denuncias de violación a los derechos humanos sucedidas en las últimas dictaduras del siglo $\mathrm{XX}$ han significado una suma de nuevos desafíos y debates al interior de diversas disciplinas sociales y jurídicas, tanto en lo que tiene que ver con las dificultades de su abordaje como en la discusión sobre el rol de los y las investigadoras en la configuración de un nuevo campo.

Las movilizaciones de las organizaciones de derechos humanos en torno a la búsqueda de la verdad y la justicia sobre estos crímenes, su 
posterior incorporación a la agenda política de los gobiernos democráticos junto con los distintos procesos de justicia, y reparación llevados adelante a lo largo de los años y hasta la actualidad han generado la necesidad de incorporar herramientas provenientes de distintas disciplinas en los procesos de investigación vinculados a esta materia. Lo anterior junto con la constante demanda proveniente de distintos sectores de la sociedad civil referida a una investigación de mayor alcance, profundidad y eficacia con respecto al tema nos presenta el reto de realizar una sistematización y puesta al día de los avances y novedades metodológicas producidas a partir de la experiencia propia en el campo y del entrecruzamiento disciplinar.

El presente artículo trata sobre el proceso de construcción de un abordaje metodológico desde las ciencias sociales y humanas enmarcada en el enfoque transdisciplinar, que se origina a partir del trabajo realizado durante más de quince años en la investigación sobre los crímenes cometidos por el estado uruguayo dentro de su territorio y en el marco del Plan Cóndor. Esta propuesta metodológica pretende brindar las herramientas necesarias para poder llevar adelante investigaciones de corte académico que tengan entre sus propósitos contribuir a los procesos de verdad, justicia y reparación en relación con las graves violaciones a los derechos humanos cometidas en las últimas dictaduras del siglo XX en el Cono Sur. El contenido del artículo forma parte de un proyecto más amplio destinado a generar un campo de estudio en la materia que permita seguir avanzando en la construcción de estas herramientas.

\section{Las violaciones a los derechos humanos en el pasado reciente uruguayo: las investigaciones históricas y su judicialización.}

Entre el 27 de junio de 1973 y el 1 de marzo de 1985, Uruguay vivió bajo una dictadura reconocida por su carácter cívico-militar. Previo a eso, y desde 1968, el país había transitado un proceso de avance del autoritarismo con la aplicación sistemática de medidas de excepción, conocido como "el camino democrático a la dictadura" (Rico, 2005). La 
estrategia represiva principal diseñada por el estado uruguayo ya desde los '60 fue la utilización de la prisión prolongada y la tortura, existiendo, además, casos de desaparición forzada (sobre todo en el marco de la coordinación represiva regional - Plan Cóndor) y casos de asesinato y fallecimiento por responsabilidad y/o aquiescencia del Estado. Aproximadamente 25.000 ciudadanos y ciudadanas sufrieron algún tipo de detención y tortura, en tanto fueron procesados por la justicia militar y sufrieron la prisión política cerca de 6000 personas (Rico et al., 2008). Por otra parte, hasta el momento se tiene conocimiento de 197 casos de desaparición forzada y 202 casos de asesinato político (EIH, 2019).

La transición democrática pos dictadura (1985-1989) estuvo marcada por la discusión sobre cómo abordar los crímenes cometidos por el Estado en el pasado reciente. En ese período diversas acciones se llevaron a cabo, unas con el objetivo de clausurar el tema y otras buscando avanzar en la verdad y la justicia (Figueredo y Larrobla, 2019), pero no fue sino hasta el año 2000 cuando la temática fue asumida por el gobierno de ese momento ${ }^{1}$ conformando la Comisión para la Paz, la que cumplió el rol de las Comisiones de Verdad que antes se habían instalado en otros países (Figueredo y Larrobla, 2019).

Los cambios más significativos con respecto a la gestión de este tema se procesaron a partir de que la coalición progresista Frente Amplio asume por primera vez el gobierno, el $1^{\circ}$ de marzo del 2005. Dos días después, el 3 de marzo, se firmó un Acuerdo General de Cooperación entre Presidencia de la República y la Universidad de la República (Udelar), suscribiendo un convenio específico sobre el tema "Derechos Humanos y detenidos-desaparecidos". El mismo estableció que la Universidad aportaría los equipos de investigación, constituyéndose así los equipos de Antropología y de Historia, radicados en la Facultad de Humanidades y Ciencias de la Educación (FHCE). El primero tendría a cargo las excavaciones en busca de los restos de las personas desaparecidas y el segundo la revisión documental y la redacción de un informe. La gran novedad de este convenio radicó en que era la primera vez que un gobierno

1 Nos referimos a la administración del Partido Colorado, encabezada por el Dr. Jorge Batlle. 
encomendaba a equipos universitarios la investigación sobre los crímenes cometidos por el Estado en su pasado reciente (Larrobla y Larrobla, 2016).

El equipo de historiadores ${ }^{2}$, que conformamos, comenzó su labor en setiembre de 2005 y culminó el 4 de junio de 2007 con la presentación por parte del Presidente de la República, Dr. Tabaré Vázquez, de los 5 tomos de las investigaciones históricas (4 tomos) y arqueológicas (1 tomo) sobre detenidos-desaparecidos. Ese primer informe de carácter histórico se estructuró en torno a dos ejes principales: la dimensión individual de la víctima de desaparición forzada, a partir de la elaboración de fichas personales de cada una de ellas y el contexto histórico en que sucedieron las mismas, presentando los operativos represivos contra las organizaciones políticas en que se enmarcaron esas desapariciones, entre otras secciones. Para esto se realizó un trabajo de recopilación y selección documental, ordenando, relacionando y entrecruzando información con el objetivo de recomponer la lógica de los hechos desde una perspectiva histórica.

Por primera vez accedimos a archivos estatales que contenían documentación producida por el Estado en dictadura y el encuentro con ésta produjo un momento de perplejidad; ante lo desconocido fuimos invadidos por un sinnúmero de emociones, como si nos encontráramos frente a un otro con el que no compartimos un mismo universo de significados (Guber, 2011: 47). Esta perplejidad se acompañaba, a su vez, por una sensación de premura constante vinculada a los tiempos acotados establecidos para la finalización de la investigación ${ }^{3}$. El análisis de estos dos elementos será de vital importancia en el futuro de las investigaciones, pero en este contexto de los años 2005-2007 los objetivos estaban dirigidos a poder realizar una primera investigación que brindara herramientas para continuar avanzando y profundizando en la reconstrucción de la verdad:

\footnotetext{
2 El equipo recibió esa denominación teniendo en cuenta que en ese momento sus integrantes eran historiadores, a excepción de una de ellas, que es Lic. en Ciencia Política. Posteriormente modificó su nombre a Equipo de Investigación Histórica.
}

3 El convenio establecía a su inicio 6 meses de trabajo de investigación. Posteriormente se realizaron sucesivas prórrogas que llegaron hasta el año 2007. En la segunda administración progresista el equipo de investigación fue nuevamente convocado a continuar con las investigaciones. 
Lo que sigue a continuación es una investigación histórica sobre detenidos desaparecidos. (...) hemos tratado de abstenernos de realizar interpretaciones teóricas o explicitar nuestras conclusiones acerca del terrorismo de Estado y las violaciones a los derechos humanos en Uruguay, sus causas históricas, sus responsables y sus efectos en el presente. (...).

Lo importante en clave ciudadana era presentar un estudio serio acerca del esclarecimiento de acontecimientos especialmente trágicos que signan el período estudiado. Se trata, en suma, de aportar una obra documentada que contribuya a esa necesaria reflexión y discusión colectivas que la propia sociedad uruguaya seguirá procesando por distintos canales $(\ldots)^{4}$.

La investigación tuvo desde el comienzo objetivos amplios que buscaron contribuir al conocimiento del terrorismo de Estado. Su carácter de informe, asociado a lo que fueron otros informes previos en la región, como el Informe de la Comisión Nacional sobre la Desaparición de Personas - CONADEP - de Argentina (1984) o el Informe de la "Comisión Nacional de Verdad y Reconciliación" en Chile (1991) le otorgó ciertas particularidades en enfoques y abordajes y le dio características generales a una investigación que se basó fundamentalmente en los mecanismos y dispositivos represivos en Uruguay y en la región. Asimismo y desde el punto de vista historiográfico, recibió influencias tanto de la microhistoria 5 como de la historia política6 las que han tenido una fuerte incidencia en la

\footnotetext{
4 Investigación Histórica sobre detenidos- desaparecidos en cumplimiento del artículo $4^{\circ}$ de la Ley de
} Caducidad, Tomo 1, Montevideo, IMPO, 2007. Pág. 34.

5 De la microhistoria "porque se ha concentrado en el estudio de la experiencia de los sujetos, (...) donde la presencia de los actores de esa historia exige la utilización de nuevas herramientas de trabajo y donde la falta de distancia temporal indica la necesidad de un análisis en pequeña escala y una observación minuciosa". FRANCO, Marina y LEVIN, Florencia. "El pasado cercano en clave historiográfica", en: 
emergencia de la historia reciente. (Franco y Levin: 2007, 5-6). De esta manera, la metodología aplicada en esa primera etapa se apoyó en la historiografía tradicional, que comprende una investigación descriptiva, fundamentada en el orden cronológico de los acontecimientos y centrada en el trabajo documental.

Además, nos focalizamos en la sistematización de la documentación recopilada. Recordemos que era la primera vez, luego de años de democracia, que se accedía a varios repositorios documentales (hasta el momento ni conocidos ni abordado por investigadores) y era la primera vez también que éstos entraban en diálogo con otros.

Lo testimonial - a diferencia de las investigaciones de las Comisiones - fue incorporado para relatar, corroborar y apoyar algunos relatos históricos, no ocupando un lugar central. De esta forma incluimos, en su gran mayoría, testimonios (entrevistas en algunas ocasiones) obtenidos en otras circunstancias históricas. Solo en casos puntuales realizamos entrevistas con fines específicos, dirigidas principalmente a chequear algún dato o reconstruir fragmentos de contextos represivos. La publicación de los 5 tomos conteniendo los resultados de la investigación generó un antes y un después en el campo de estudio sobre el pasado reciente, erigiéndose como una obra ineludible y de primera referencia para cualquier investigador que pretenda abordar este campo.

El proceso de investigación, por tanto, no culminó con la publicación sino muy al contrario fue un recorrido que intercaló descubrimientos documentales, preguntas e interpelaciones sobre su contenido, sobre sus productores, sus contextos y que generó debates internos referidos a la metodología de abordaje. Estas discusiones no

FRANCO, Marina y LEVIN, Florencia (compiladoras), Historia reciente. Perspectivas y desafíos para un campo en construcción, Buenos Aires, Paidós, 2007. Pág. 6.

6 De la historia política porque "junto con la nueva importancia otorgada al "acontecimiento" en el último tercio del siglo $\mathrm{XX}$, ha sido un factor estrechamente ligado a la emergencia de la historia reciente, tan vinculada a los hechos de la Segunda Guerra Mundial. A su vez, la reaparición de esta mirada política en el campo historiográfico está relacionada con el espacio explicativo que ella concede al factor de la contingencia y a la dimensión individual como elementos del análisis histórico (Rousso, 2000), como así también al interés por el estudio de las representaciones y los imaginarios sociales". Ídem. 
tomaron en cuenta la futura judicialización de las investigaciones, cuestión que no estuvo prevista ni imaginada en esa primera etapa de trabajo.

Durante la segunda administración del gobierno progresista a cargo de José Mujica (2010-2014) volvimos a ser convocados como Equipo de Investigación Histórica (EIH) para continuar con las investigaciones sobre el período. En esta segunda etapa se ampliaron los cometidos y se incorporó el período previo a la dictadura (1968-1973) y la dimensión del asesinato político. El año 2011 se inició con el conocimiento de la Sentencia dictada por la Corte Interamericana de Derechos Humanos (22 de febrero), en el caso Gelman contra el Estado uruguayo la que planteaba omisiones e incumplimientos por parte de Uruguay en referencia a las violaciones de los derechos humanos en su pasado reciente. Ésta ocasionó diversas tensiones en el ámbito político en la medida en que el gobierno intentó dar cumplimiento con las recomendaciones de la Sentencia. En ese marco, el 30 de junio se dictó el decreto 322/011 que revocó "por razones de legitimidad los actos del Poder Ejecutivo que habían impedido la investigación de denuncias" (Castro, 2018) y el 27 de octubre de ese mismo año, luego de largas jornadas de discusión parlamentaria, se aprobó la Ley 18.831 "que restableció el pleno ejercicio de la pretensión punitiva del Estado". (Castro, 2018), calificando a los delitos cometidos por éste como crímenes de lesa humanidad a los que no se les debería computar el plazo de prescripción.

Bajo esas circunstancias y con un nuevo escenario legal, la justicia penal comenzó a requerir nuestros conocimientos técnicos a través de oficios judiciales que solicitaban información y documentación vinculadas a denuncias sobre crímenes de lesa humanidad. Es así que desde fines del 2011 y principios del 2012 las investigaciones históricas y la documentación que fue anexada en ese primer informe (2007) comenzó a ser demandadas por la justicia para ser incorporadas en las distintas causas tramitadas en Uruguay y también en la República Argentina, sobre desaparición forzada, asesinato político y/o prisión prolongada.

Al inicio las solicitudes judiciales fueron respondidas exclusivamente con la documentación hallada, sin que se adjuntara ninguna explicación sobre su contenido ni sobre el contexto; pero rápidamente asumimos que el aporte fundamental no era sólo el documento sino lo que se podía decir de él y en eso radicaba su relevancia. Resolvimos, entonces, acompañar la documentación con la confección de informes técnicos que le dieran sentido y significación a lo anexado. Acompañando esta primera 
resolución, la siguiente fue que daríamos respuesta a todas las solicitudes, aún aquellas que referían a la prisión política, dimensión ésta que no estaba incluida dentro de los cometidos que se nos asignaron y que por tanto la documentación encontrada en su momento contaba con bajos o nulos niveles de sistematización.

Por otra parte, comenzamos a ser convocados como testigos calificados en los juicios en curso, lo que significó un redimensionamiento de la tarea llevada adelante y una profunda transformación del trabajo. En ese marco de cambios, comenzó el proceso de construcción de un nuevo abordaje metodológico que nos permitiera dar cuenta del nuevo rol que cumplían las investigaciones en el avance de las causas penales y en los procesamientos de los responsables de los delitos.

En ese contexto, a su vez, las organizaciones defensoras de los derechos humanos y diversos activistas comenzaron a demandar una investigación enfocada a la resolución de problemas vinculados a la posibilidad de realización de justicia, reparación y memoria, generando al interior del equipo y también en el marco universitario (aunque más tarde en el tiempo) una serie de intercambios que son reflejo del debate historiográfico sobre el papel de los historiadores y su vínculo con la Justicia. Entendemos que esta discusión se enmarca en una serie de debates que se pueden situar a partir de la década de los '80, que incluyen la temática de la memoria y el lugar del testimonio y el testigo, originados a su vez en el "desplazamiento del centro de gravedad de la historia hacia lo contemporáneo" (Nora, 2018). En el recorrido del mismo puede verificarse, a partir de la necesidad de establecer las diferencias entre el oficio del historiador y del juez y las verdades que ambos pretenden producir, la fuerte interpelación que supuso para los historiadores el abordaje de acontecimientos ubicados en la historia reciente, en un contexto donde la justicia los convocaba o bien como testigos o a través de la inclusión de investigaciones históricas en los procesos judiciales.

Es así que autores como Ginzburg en su ensayo "El Juez y el Historiador" (1993) o Tzvetan Todorov en "Memoria del mal, tentación del bien" (2002), reflexionan en profundidad sobre las relaciones entre las disciplinas histórica y jurídica, sus metodologías de análisis o el modo en que utilizan la prueba (Ginzburg: 1993) o las diferencias entre la verdad histórica y la jurídica (Todorov: 2002). Ricoeur, por su parte, sintetiza claramente el origen de las tensiones entre ambas disciplinas al señalar que "la incriminación jurídica descansa en el principio de la culpabilidad 
individual" en tanto que "el historiador extenderá su investigación a un número mayor de actores (...) en el marco de encadenamientos más amplios, más complejos” (Ricoeur, 2004: 423), y por tanto cuando el proceso judicial requiere ampliar los niveles de análisis "el juez pasa la palabra al historiador" (Ricoeur, 2004: 425). Esa ampliación necesaria y fundamental sucede, generalmente, cuando los juicios llevados adelante tratan sobre crímenes colectivos cometidos por los Estados contra sus propias poblaciones u otras. Conceptos tales como objetividad y distancia del investigador (Hartog, 2001), se vuelven centrales en estos intercambios, en momentos en que además se procesan cambios epistemológicos a nivel de las ciencias sociales.

Es en el Cono Sur, en el ámbito intelectual de Argentina, que se producen diferentes intercambios que problematizan y reflexionan con respecto a la presencia del académico en la justicia. Tomaremos la perspectiva de Gabriela Águila (2014) quien señala que los abordajes sobre el pasado reciente no tienen, hoy en día, como única protagonista a la disciplina histórica, al contrario, actualmente es un espacio en el que se encuentran y dialogan varias disciplinas. Asimismo, los cruces y articulaciones entre los recorridos judiciales y los desarrollos de las ciencias sociales en general se han vuelto cada vez más visibles y constantes. Es así que fiscales y jueces

recurren a algunas producciones del campo académico para sostener sus argumentaciones, muchos historiadores y cientistas sociales han sumado a su quehacer profesional el trámite de diversas causa por violaciones a los derechos humanos, participando como peritos, testigos, aportando sus textos como fundamento de sentencias, etc. (Águila, 2014: 25).

Este entrecruzamiento no pretende significar que la justicia y la historia tengan los mismos fines, en tanto esta última tiene como objetivo explicar y comprender el pasado y no juzgarlo (Águila, 2014: 25), coincidiendo en este caso con lo planteado por los autores mencionados anteriormente. También desde Argentina, pero esta vez desde la 
antropología, Santiago Garaño nos acerca una mirada diferente, distanciada de las interpelaciones y dudas provenientes de la disciplina histórica en cuanto a la vinculación con la justicia. En su artículo "El conocimiento antropológico en el marco del Proceso de Memoria, Verdad y Justicia: Reflexiones sobre una experiencia como "testigo de contexto" en el marco del Juicio "Operativo Independencia (primera parte)" (2018) se vislumbra otra forma de repensar los perfiles profesionales desde un enfoque innovador. A partir de una experiencia personal "reflexiona sobre una nueva figura que ha ido ganando presencia en los juicios sobre crímenes de Lesa Humanidad, que es la del "testigo de contexto". El "testigo de contexto" es aquel especialista, generalmente proveniente de las ciencias sociales, "que es presentado en calidad de testigo y cuyo aporte es tomado como prueba testimonial". (Garaño, 2018: 2). Ser "testigo de contexto" implica -entre otras cosas- hacer comprensible e inteligible lo elaborado en las investigaciones de forma tal de poder explicarlo ante una audiencia compuesta por público diverso (jueces, abogados, asistentes) pero sin perder "la legitimidad del experto". (Garaño, 2018: 3 y 4).

En Uruguay la polémica sobre la relación entre la disciplina histórica y la jurídica no tuvo mayor visibilidad pública y se expresó tangencial y tardíamente en dos artículos que fueron publicados en el año 2016 en la Revista Contemporánea7. El título de la polémica era "Situación de los archivos del pasado reciente" y los artículos fueron escritos por Vania Markarián ${ }^{8}$ ("Los documentos del pasado reciente como materiales de archivo. Reflexiones desde el caso uruguayo") y Álvaro Rico ${ }^{9}$ ("El actual malestar de los historiadores: entre la defensa del oficio y la responsabilidad política. Sobre archivos y repositorios documentales").

Markarián, a partir de un recorrido sobre el proceso uruguayo de los últimos 10 años y la situación de la documentación sobre el pasado reciente en el marco de ese proceso, dedicó un espacio importante a la Investigación Histórica sobre Detenidos- Desaparecidos, realizada por el

7 La Revista Contemporánea es una revista que surge en el 2010 dirigida a quienes realizan estudios históricos. Es coordinada por tres historiadores: Vania Markarián, Aldo Marchesi y Jaime Yaffé. (Todos en algún momento miembros del Equipo de Historiadores).

8 Vania Markarián formó parte del equipo de historiadores entre el 2005 y el 2007.

9 Álvaro Rico fue el coordinador del equipo de historiadores durante 10 años. 
equipo de historiadores y publicada en el año 2007, deteniéndose en las relaciones de los historiadores con la justicia. La historiadora entiende que estos libros "empezaban a armar un puzzle de voces y miradas diversas". Y que los mismos "equiparaba de hecho los métodos clásicos de la investigación histórica erudita en archivos con la producción de evidencia, aunque fuera contextual, para los juicios que se estaban reabriendo en el nuevo contexto político" (Markarián, 2016: 185-186), lo que derivó en la inclusión prácticamente sistemática de estos trabajos en demandas y sentencias, mostrando que el esfuerzo por atender los reclamos de las víctimas había primado en los criterios de detección y búsqueda de repositorios (Markarián, 2016: 186). A su vez señala lo que para ella constituye un riesgo y es que los investigadores no logren distanciarse de sí mismos, en tantos ciudadanos demandantes de verdad y justicia:

En tanto ciudadanos [refiriéndose a los investigadores], sus demandas pueden coincidir con las de quienes reclaman "verdad" y "justicia" (y en Uruguay lo han hecho casi sin excepciones), en tanto practicantes de una disciplina con sus propias pautas de legitimidad, criterios de verdad y métodos de construcción de pruebas, sus urgencias y premisas de trabajo son de naturaleza diferente. Habitar las dos pieles y ocasionalmente poner una al servicio de la otra, con inequívoca, vocación de justicia, no borra esa distinción radical. (Markarián, 2016: 186).

Álvaro Rico, responde a estos dichos señalando, en primer lugar, que la mirada de Markarián implica al mismo tiempo un reconocimiento y una subestimación:

El fondo del problema que, a mi modo de ver, plantea Vania, es acerca del verdadero valor cognoscitivo y aporte historiográfico de las investigaciones históricas (...) a partir de una disyunción planteada por la articulista que, por un lado, reconoce su aporte desde el punto de vista ciudadano y, por otro lado, lo subestima desde el punto de vista historiográfico porque, fundamentalmente, las reglas del oficio del historiador no son las de aportar pruebas a la Justicia entre otras razones. (Rico, 2016: 209). 
En segundo lugar, aclara que las investigaciones se judicializan una vez concluidas las mismas y que "la judicialización no fue un "investigar para la Justicia" (Rico, 2016: 209), señalado que los investigadores no realizaron la investigación en calidad de peritos de la justicia, sino que es la justicia la que las incorpora en sus instancias procesales. Hacia el final cuestiona si existe una "verdad jurídica" distinta de una "verdad histórica", a lo que responde "creemos que no, pero hay que demostrarlo. En todo caso, la verdad de los hechos del pasado reciente no solo es parte de la búsqueda permanente del historiador sino la base de una noción de justicia como virtud y de decisiones judiciales justas" (Rico, 2016: 210).

Este debate Rico-Markarián atravesó los intercambios que realizamos al interior del equipo con anterioridad a su publicación, aunque no fue sino hasta el año 2015, coincidiendo con la nueva administración progresista, que ese proceso comienza a cristalizar en el diseño de una nueva estrategia que diera cuenta de las transformaciones y cambios operados a partir de lo demandado por la justicia al equipo de investigación. En esta posibilidad de avanzar en las reflexiones sobre lo realizado hasta el momento, sus alcances y su necesidad de transformación influyeron diversos eventos.

En primer lugar, el equipo modificó su integración, con la incorporación de investigadoras provenientes de la Antropología y la Sociología10, y debido a cambios en la gestión de la Universidad ${ }^{11}$, la coordinación del mismo quedó a cargo de Fabiana Larrobla, proveniente de la Ciencia Política.

En segundo lugar, la interacción con la justicia se intensificó y nos obligó a pensar más allá de las disciplinas de origen de cada integrante donde uno de los mayores desafíos estuvo representado por la necesidad de hacer inteligible para otros ajenos al campo de estudio, los episodios del

10 En la actualidad la integración del equipo es la siguiente: Fabiana Larrobla (coord.) Politóloga, Magdalena Figueredo, Historiadora, Graciana Sagaseta, estudiante avanzada de Historia, María del Carmen Martínez, activista de derechos humanos, integrante de la organización Madres y Familiares de Uruguayos detenidos-desaparecidos, Isabel Cedrés, Politóloga, Paula Duffour, estudiante avanzada de Sociología, Juana Urruzola, estudiante avanzada de Antropología.

11 En agosto de 2014 resultó electo Rector de la Universidad de la República el matemático Roberto Markarián. Su oponente en las elecciones universitarias había sido Álvaro Rico. 
pasado que eran y son juzgados en el presente. Estos episodios, además, debían necesariamente ser enmarcados en un contexto histórico determinado para poder ser comprendidos, ya que no hablamos de crímenes individuales, aunque la justicia los aborde como tales, sino de delitos cometidos por el propio Estado contra sus ciudadanos. Lograr articular todas estas dimensiones (contextuales, individuales, históricas, políticas, etc.) y traducirlas en un relato comprensible requirió un importante esfuerzo, en la medida en que, por ejemplo, el lenguaje que tradicionalmente habíamos utilizado debió ser ajustado. En este sentido, es relevante señalar la coincidencia con lo planteado por Garaño en el artículo mencionado anteriormente.

En tercer lugar, y configurando otro de los grandes desafíos, la necesidad de construcción de un respaldo documental sólido y consistente que sustentara las hipótesis y afirmaciones que realizamos en los informes, los que además se transforman en elementos de prueba que los actores jurídicos utilizan en las audiencias.

Esta construcción, a su vez, implicó una revisión de lo realizado hasta el momento, prestando atención a detalles que antes no parecían relevantes y, en este sentido, surgió la necesidad de poder establecer espacios de trabajo conjunto con otros actores que pudieran aportar su conocimiento en base a la vivencia como víctimas.

En este marco fueron sucediendo los cambios y las tradicionales herramientas metodológicas se adaptaron al nuevo contexto de producción del conocimiento, a la vez que se sumaron otras, en la comprensión de que los procesos de investigación son dinámicos, abiertos y creativos, y que "requieren variedad de posibilidades, de técnicas y de instrumentos acordes con las múltiples situaciones que se pueden presentar a lo largo de la indagación”. (Hurtado de Barrera, 2008).

La nueva perspectiva requirió dar cuerpo teórico a nuestras intuiciones, que habían sido generadoras de rápidas respuestas a requerimientos que así lo exigían y con los que, además, nos sentíamos profundamente comprometidas. En ese recorrido que puede definirse como experimental es que nos acercamos al enfoque transdisciplinar. 


\section{Nueva mirada en la investigación: el enfoque transdisciplinar.}

La nueva forma de producción del conocimiento implica reconocer la complejidad de los procesos a investigar y por tanto la imposibilidad de abordarlos sólo a partir de una mirada disciplinar. En nuestro caso, la propia conformación de un equipo de investigación donde convergen distintas disciplinas, y cuyos integrantes somos capaces "de colocar nuestro saber e intenciones en función del tema investigado" (De la Herrán, 2011), produjo una forma de trabajo en donde las fronteras disciplinares se transformaron en puentes de comunicación y cooperación. El intercambio constante y la puesta en común de perspectivas diversas produjo redefiniciones a medida que el proceso de investigación avanzaba, modificando las miradas y creencias que hasta ese momento se sostenían. El equipo se expuso y se expone, así, a vivir la experiencia de este proceso permitiendo que éste lo transforme (De la Herrán, 2011).

La mirada profesional por tanto no realiza un sólo recorrido (desde la singularidad de su disciplina) sino que integra y fusiona abordajes que provienen de la experiencia acumulada y de romper con ciertos límites y prejuicios. Cuando se nos presenta una porción del todo, una fracción del pasado, la mirada se posa desde una perspectiva que percibe y analiza de un modo global, que realiza conexiones para obtener una visión de conjunto de un sistema complejo.

Por otra parte, este enfoque supone la producción de conocimiento buscando resolver problemas (en nuestro caso: responder solicitudes de la Justicia Penal, por ejemplo), dando fin a la distinción entre investigación "básica" e investigación "aplicada", ya que la propia dinámica del proceso habilita y estimula la circulación del conocimiento en los distintos niveles sin que sea necesario utilizarla (Aronson, 2003).

Por sus características este nuevo abordaje incluye la interacción y diálogo con otros actores, no necesariamente provenientes del campo académico, los que participan enriqueciendo el proceso con sus propios saberes y conocimientos. En este sentido hay un acercamiento a la investigación acción-participativa (García, 2006), en la medida en que la integración de la población afectada - en este caso por el terrorismo de Estado - es imprescindible. 
En nuestro caso, y como ya señalamos anteriormente, este enfoque fue gestándose desde la propia práctica y frente al desafío que representa la pregunta de cómo avanzar en el campo de la verdad y la justicia a partir de las investigaciones. Para dar cuenta de estas transformaciones en la propia práctica presentamos un recorrido sobre el proceso de producción que culmina con la confección de un informe técnico como síntesis de los resultados obtenidos en ese trayecto. Si bien los distintos elementos que lo componen se registran por separado esto es sólo a los efectos de facilitar su descripción, pero entendiendo que en la práctica forman parte de un mismo entramado, donde se cruzan de diversas formas. Por otro lado, el propio tipo de abordaje supone la flexibilidad en los procesos, lo que significa que en ocasiones es necesario sumar otros elementos y herramientas a la investigación.

\section{El proceso de investigación y el análisis de los distintos elementos.}

Anteriormente señalamos que el trabajo llevado adelante por el equipo, desde sus inicios, se basó fundamentalmente en documentación producida por distintas agencias e instituciones del Estado bajo el período de estudio. La novedad consistió en el acceso a archivos con documentación producida por agencias represivas y es a esta novedad que nos vamos a referir12, ya que es la que ha presentado mayores dificultades en el análisis y abordaje.

La discusión generada en cuanto a cómo abordar esa documentación al mismo tiempo llevó implícita la interrogante de cómo comprender su orden en archivos producidos con una lógica desconocida

12 En el año 2006 se accedió a la entonces Dirección Nacional de Información e Inteligencia (DNII), actualmente Dirección General de Información e Inteligencia, dependiente del Ministerio del Interior. En el 2019 se tuvo acceso a documentación proveniente del Servicio de Información de Defensa, dependiente de la Junta de Comandantes en Jefe y en el 2016 se accedió al archivo del Cuerpo de Fusileros Navales (FUSNA) de la Armada Nacional, otra agencia que cumplió un rol importante en cuanto acciones de inteligencia y represión del período. 
para los investigadores, lo que devino inexorablemente en comprender al propio archivo en el que se intervenía. Esto derivó, a su vez, en comprender al agente productor, que a la vez era el agente represor, su dinámica y consistencia interna. Este recorrido guiado por la necesidad de comprender un universo que nos era ajeno fue realizado en varios planos en forma simultánea, porque no se trataba de comprender algo para luego comprender lo otro, sino de que las comprensiones y los desciframientos en una dimensión nos trasladaban a otra dimensión, en un trayecto no lineal, sino de múltiples cruces.

Desde el primer contacto con un documento producido por una agencia de inteligencia hasta la actualidad recorrimos un largo camino en el aprendizaje sobre cómo dar lectura a los mismos. No sólo importa el contenido, lo que dicen u omiten, sino la forma en que se estructuran, la codificación que utilizan, los niveles de confiabilidad que la misma agencia adjudica a la información que se difunde, la identificación de los sellos que se estampan, los niveles de reserva del mismo, etc.

Asimismo, los documentos dialogan con el contexto, con lo que acontece en el momento de producción de éstos, pero también con lo que acontece en el momento en que son reactualizados y archivados junto con otros (Jelin, 2002: 1-12). El diálogo que se establece entre documento y contexto es complejo, porque forma parte de una red donde el "ir" y "venir" puede ser imaginado más como trazo que va dibujando distintos diagramas y figuras que como una línea que tiene un principio y un fin.

Colocar en esa trama al documento y acompañarlo en el recorrido que realiza permite ir iluminando las zonas que se mantenían ocultas. Los recorridos documentales en estos casos responden a una lógica (propia de las agencias de inteligencia) que requiere ser develada. En ese trabajo de vincular y dar sentido a ese aparente caos documental es que comenzamos a construirse la evidencia que permitirá trascender esa lógica del secreto y clandestinidad en que se instrumentaron las acciones represivas del Estado. Existen diversos tipos documentales que dan cuenta de las distintas formas de organizar la información y de diseminarla (utilizando un término propio de estos organismos), poder identificarlos es el primer paso para acercarnos al mismo. Mencionaremos sólo algunos de ellos a modo de ejemplo: fichas patronímicas, Partes Especiales de Información, Memorándums, Partes de Novedades diarias o mensuales de Información, Informes de Agente, etc.

A su vez cada tipo de documento cuenta con una estructura documental propia, que por lo general se repite en todas las agencias, con 
algunas variaciones menores. Los encabezados nos señalan el organismo productor del documento y su dependencia jerárquica, así como la fecha y hora de confección, en muchos de ellos, en general en los Partes Especiales de Información (P.E.I.) nos encontramos con un código que evalúa la confiabilidad de la información y de la fuente que brindó la información. Al final del documento se listan las agencias entre las que será "diseminada" la información. También podemos encontrar otros elementos: sellos que acusan recibo o que señalan que fue archivado y sus correspondientes fechas.

Todo esto nos permite no sólo aproximarnos a la comprensión del documento en sí, sino también al funcionamiento de cada agencia que lo produce, los departamentos que la integran, los responsables que lo firman, su grado y su función. En nuestra experiencia de trabajo muchas veces ha sido a partir de este primer análisis que hemos ido reconstruyendo las estructuras de los órganos represivos del Estado.

Cuando nos enfrentamos a la lectura de un documento de estas características puede existir la tentación de suponer que lo que está escrito o es verdad o es mentira, nuestros propios prejuicios inconscientes pueden llevarnos a realizar una lectura que quede atrapada en esa dualidad de "mentira" y "verdad", por tanto es necesario ejercitar un primer distanciamiento (Elías, 1990), ya que el contenido de los mismos es integrante de esa verdad que pretendemos construir aun sabiendo que puede ser una verdad que miente sobre la realidad o los hechos ocurridos. El abordaje requiere ir más allá de lo escrito, reconociendo la existencia de vacíos y omisiones (Da Silva Catela, 2002: 397) que se evidencian a partir del entrecruzamiento realizado con otras fuentes documentales y testimonios y del conocimiento adquirido sobre el funcionamiento de las agencias y el lenguaje utilizado por las mismas. (Da Silva Catela, 2002: 391). Distinguir los montajes artificiales para que un crimen quede impune, así como reconocer un informe que brinda datos certeros, es un trabajo que nos implicó tomar posesión del lugar del otro: animarnos a adentrarnos en un territorio desconocido donde existe un universo de significados para comprender y aprehender.

Son cientos los documentos dispersos en distintos archivos, que se agrupan con una lógica incierta y que muchas veces pueden parecer irrelevantes para quien accede por primera vez a ellos. El ejercicio de hacerlos hablar nos lleva a establecer una estrategia de análisis que supone incorporarlos a una red de múltiples diálogos con otros elementos para 
luego realizar el recorte que permita observar y estudiar el detalle desde diversas perspectivas, volviendo a recolocarlos en ese lugar donde comienza a operar el sentido que en el pasado se mantenía oculto. En esta dinámica, el documento analizado no lo es todo, sino que es parte de un entramado al que ilumina y a la vez oscurece, generando un continuo "ir y venir" de quienes investigamos, desde una perspectiva analítica. "Cada documento informa tanto como oscurece” (Entrevista a Barrán, 2010: 186), decía el Prof. José Pedro Barrán, hablando sobre la tarea del investigador. Cada documentación hallada conecta no sólo con lo visible sino también con todos los espacios invisibles.

Una de las claves para evaluar la información contenida en los documentos es la conciencia de saber que estamos trabajando con materiales producido por un Estado que operaba en secreto y que a la vez continuaba sosteniendo el andamiaje público y burocrático que le brinda continuidad. También es clave conocer qué dependencias y organismos cumplen con esos cometidos de construir la comunicación pública y la comunicación que denominamos "secreta" y en qué momentos cumplían con un cometido u otro, ya que la acción estatal que se despliega en ambas vías, produce el cruzamiento de sus dependencias hacia una o hacia otra según las circunstancias.

Perpetuar los crímenes y prolongar la impunidad a través del tiempo es un trabajo que requirió de la participación combinada de muchos actores y organismos, desmantelar ese montaje requiere por tanto conocer en profundidad el desarrollo y evolución de esa dinámica. Un ejemplo interesante y contundente de este eje "verdad" y "mentira" lo constituye la lectura simultánea de informes secretos confeccionados para que circulen al interior de las agencias y las respuestas producidas por el Ministerio de Relaciones Exteriores ante las demandas de los organismos internacionales, todo en relación al mismo caso. Trabajar desde esta perspectiva contribuye a darle forma y solidez a documentos de otra manera es posible que no la tuvieran (Ginzburg, 1993: 3-4).

En lo que tiene que ver con la incorporación de lo testimonial en la investigación, partimos del conocimiento de que es una de las fuentes que ha generado múltiples polémicas en cuanto a su utilización, pero aun así sigue siendo medular e ineludible en las investigaciones dedicadas a las violaciones de los derechos humanos en el pasado reciente. Beatriz Sarlo, una de las más crítica en esta área, cuestiona su carácter preponderante y a veces principal en los relatos y alerta enfáticamente que "no son la única 
fuente de conocimiento" (Sarlo, 2005: 63). En ello hay bastante consenso ya que el testimonio casi nunca está solo, sin embargo, es cierto que existen divergencias en torno a su significado, preponderancia y sobre lo que genera y aporta a la narración histórica. Sarlo cuestiona la utilización del testimonio en el relato histórico aunque entiende su trascendencia en el campo de la justicia. Proponiendo y planteando en contrapartida el uso de fuentes escritas de la época, como elemento destacado.

Alejandra Oberti, Elizabeth Jelin y Pilar Calveiro, sostienen sin embargo otras posturas. Calveiro, citando a Jelin, recuerda un aspecto fundamental que no debe perderse de vista, al momento de abordar las fuentes: "ningún texto puede ser interpretado fuera de su contexto de producción y de su recepción, incluyendo las dimensiones políticas del fenómeno" (Calveiro, 2006: 80). Los documentos escritos de época, sobre todo si nos referimos a los producidos en la década de 1970, no constituyen elementos suficientes para abordar la complejidad de los hechos de aquel entonces. La palabra escrita parecería tener un valor "ético y puro", del que en realidad carece, como si no pudieran estos mismos ser manipulables u objeto de otras intencionalidades que distarán "de la realidad". En este sentido, los mismos, deben ser interpretados, deben ser leídos más allá de sus contenidos, y puestos por tanto en diálogo con otro tipo de fuentes. Desde esta perspectiva, y "para un conocimiento más agudo - apunta Oberti -, la lectura de los materiales producidos en el pasado necesita de la vitalidad del testimonio" (Oberti, 2009: 13).

Por otro lado, el testimonio, comprende otro elemento interesante, que Calveiro menciona con precisión, se reconoce a sí mismo como "un relato incompleto", por lo que busca obsesivamente cualquier dato que pueda ser significativo, más allá de su valoración. De esta forma, "intenta" exponer "todo", aunque ese "todo" se reconoce desde el vamos como fragmentario. El testimonio por tanto expone "su" verdad, y reclama una cierta escucha y validación social para ser parte de "la verdad" socialmente construida (por ejemplo, muchas víctimas, sintieron que su testimonio se volvió "verdadero" cuando los mismos se convirtieron en "verdad-prueba" en los juicios) (Calveiro, 2006: 77-78).

Desde nuestra perspectiva actual, el testimonio es una fuente clave y fundamental que se integra plenamente a los nuevos abordajes que están en construcción. Plegándose en pie de igualdad con todo el entramado documental se une a esta red como un eslabón más que viene a complementar y a cotejar, a preguntar e interpelar. De esta forma, contar 
con las posibilidades de resignificar memorias, recuerdos y relatos, de rever enfoques, potencia la labor de nuestra investigación abriendo cada vez más espacio y dinamiza áreas que quizás se creían "cerradas" o "acabadas".

En este recorrido que realizamos por los componentes que consideramos fundamentales en el diseño de la investigación, se encuentra el espacio de trabajo colectivo. La construcción de éste requiere de la circulación libre y sin reservas de la información y de la documentación y plantea a su vez que todos los integrantes del equipo podamos experimentar el tránsito por cada uno de los elementos que mencionamos anteriormente (archivos, análisis documental, testimonios, etc.).

El espacio de trabajo colectivo funciona en forma horizontal y es imprescindible que quienes lo integramos nos sintamos libres de plantear interrogantes, hipótesis, nuevas perspectivas de abordaje e incluso expresar sentimientos y opiniones respecto de lo investigado. Consideramos que esta posibilidad no pone en riesgo la rigurosidad de la investigación, en la medida en que podemos hacer consciente aquello que nos moviliza estamos en condiciones de saber cuánto de eso se pone en juego en el desarrollo del trabajo. Por otra parte, el espacio estimula la creación de vínculos con otros actores u organizaciones, estableciéndose intercambios diversos que aún se encuentran en una etapa muy inicial. Establecer redes flexibles que puedan encontrarse para poner en común los conocimientos acumulados y las vivencias experimentadas es un deseo y objetivo a la vez, considerando la urgente necesidad de superar la fragmentación y compartimentación que muchas veces ha caracterizado a este campo de estudio.

\section{Los resultados de la investigación. El Informe Técnico.}

Finalmente, los resultados de los procesos de investigación llevados adelante cristalizan en la confección del Informe Técnico, ya sea para ser entregado a la justicia, a las comisiones de las leyes reparatorias, o a organizaciones de derechos humanos y otros colectivos que trabajan por la memoria. El informe se acompaña de toda aquella documentación en la que se fundamentan y desarrollan las hipótesis y en todos los casos en que ha sido posible incluimos listados de oficiales policiales y/o militares que 
hayan estado en funciones en el momento y lugar en que ocurrieron los hechos investigados.

Este listado lo confeccionamos con la máxima rigurosidad y en base exclusivamente al análisis de documentación producida por el Estado (legajos personales militares, escalafones de las Fuerzas, documentación proveniente de los Ministerios de Defensa e Interior, por ejemplo), en la medida en que se ha podido acceder a ella.

Tomamos en cuenta aquello de que la justicia sólo puede condenar individuos, por tanto, la confección de estos listados reviste suma importancia porque habilita a que el proceso judicial pueda continuar su curso. Estos informes los consideramos siempre como avances de un proceso que no concluye ni se clausura en la medida en que continuamos registrando e incorporando nuevos elementos de análisis. A su vez estos retroalimentan a otros informes y a las propias fichas personales de cada una de las víctimas, las que seguimos actualizando en la medida en que se avanza en el esclarecimiento de los hechos.

\section{En suma}

Todo este proceso de investigación, con sus contradicciones, interrogantes y períodos de reflexión y debate, nos ha permitido acercarnos a reconstruir no sólo los hechos, sino el sentido de los mismos. La importancia de reconstruir el sentido está vinculada, a su vez, con el presente. Los hechos tomados como tales, aislados y sin significación corren el riesgo de quedar como relatos anecdóticos del terror. Cuando podemos dotarlos de sentido, conectarlos, vincularlos y hallar la lógica que subyace a los acontecimientos, es cuando estamos en condiciones de trabajar para evitar la repetibilidad. Porque también y en forma fundamental se trata de eso: de evitar la repetición del horror.

Este abordaje ha significado para nosotras como investigadoras una transformación profunda en lo profesional, en la medida en que hubo que despojarse de creencias previas fuertemente arraigadas en cada una y permitir ser permeadas por otros saberes, borrando los límites disciplinares que nos fragmentan y compartimentan. Dejar que las preguntas de los otros 
nos calen hondo, deconstruir lo "obvio" o "supuestamente sabido y/o evidente" y no dar por sentado nada, es uno de los constantes motores en este recorrido.

La propia experiencia nos condujo por este trayecto y en la medida que hemos podido penetrar en la densidad de la telaraña y acceder cada vez más a las zonas ocultas de las dinámicas represivas, estamos convencidas de que es por aquí que hay que seguir transitando. Esta es una de las razones principales por la que consideramos la necesidad de realizar esta primera aproximación a una sistematización de la experiencia en clave metodológica.

\section{Bibliografia}

ÁGUILA, Gabriela. "Estudiar la represión: entre la historia, la memoria y la justicia. Problemas de conceptualización y métodos", en: FLIER, Patricia (compiladora), Dilemas, apuestas y reflexiones teórico- metodológicas para los abordajes en Historia Reciente, Universidad Nacional de La Plata - Facultad de Humanidades y Ciencias de la Educación, 2014. Pp. 20-55.

ARONSON, Perla. "La emergencia de la ciencia transdisciplinar" en Revista Cinta de Moebio, Chile, número 18, 2003.

CALVEIRO, Pilar. "Testimonio y memoria en el relato histórico", en Acta Poética, 27 (2), otoño 2006. Pp. 65-86.

CASTRO, Alicia. "Derechos humanos y delitos de lesa humanidad. Un análisis sobre la jurisprudencia de la suprema corte de justicia sobre imprescriptibilidad de los delitos de la dictadura" en Revista de Derecho Público, Montevideo, año 27, número 54, diciembre de 2018. Pp. 7-34.

DA SILVA CATELA, Ludmila. "El mundo de los archivos", en: DA SILVA CATELA, Ludmila y JELIN, Elizabeth (compiladoras), Los archivos de la represión: documentos, memoria y verdad, Buenos Aires, Siglo XXI Editores, 2002. Pp. 381 - 403. 
DE LA HERRÁN, Agustín. "Complejidad y transdisciplinariedad" en "Formación Profesional", Revista Educacao Skepsis, volumen 1, número 2, San Pablo, 2011.

ELÍAS, Norbert. Compromiso y distanciamiento, Barcelona, Editorial Península, 1990.

ERLIJ, Evelyn. "Entrevista a Pierre Nora: "El historiador es un árbitro de las diferentes memorias", Revista Letras Libres, febrero 2018. Disponible en: https://www.letraslibres.com/espana-mexico/revista/entrevista-pierrenora-el-historiador-es-un-arbitro-las-diferentes-memorias

FRANCO, Marina y LEVIN, Florencia. "El pasado cercano en clave historiográfica", en: FRANCO, Marina y LEVIN, Florencia (compiladoras), Historia reciente. Perspectivas y desafios para un campo en construcción, Buenos Aires, Paidós, 2007. Pp. 1-25.

GARAÑO, Santiago. "El conocimiento antropológico en el marco del Proceso de Memoria, Verdad y Justicia: Reflexiones sobre una experiencia como "testigo de contexto" en el marco del Juicio "Operativo Independencia (primera parte)", en Sociohistórica, $\mathrm{n}^{\mathrm{o}} 41, \mathrm{e} 050$, Universidad Nacional de la Plata, Facultad de Humanidades y Ciencias de la Educación, Centro de Investigaciones Socio Históricas, Provincia de Buenos Aires, 2018.

GARCÍA, Miguel. "Un nuevo desafío en la Investigación. Enfoque transdisciplinario en Comunicación y Desarrollo" en Razón y Palabra, Instituto Tecnológico y de Estudios Superiores de Monterrey, Universidad de Los Hemisferios Quito, Ecuador, número 49, 2006.

GINZBURG, Carlo. El hilo y las huellas. Lo verdadero, lo falso, lo ficticio, Madrid, Fondo de Cultura Económica, 2010.

GINZBURG, Carlo. El juez y el historiador, Editorial ANAYA, Madrid, 1993.

GUBER, Rosana. La etnografía. Método, campo y reflexividad, Editorial Norma, Bogotá, 2001.

HARTOG, François. "El testigo y el historiador", en: Estudios Sociales. Revista Universitaria Semestral, Año XI, No 21, Santa Fé- Argentina, Universidad Nacional del Litoral, segundo semestre 2001. Pp. 11-30. 
HURTADO DE BARRERA, Jacqueline. "Investigación holística o comprensión holística de la investigación?, En: revista internacional de Magisterio, Nro. 31, 2008.

JELIN, Elizabeth. "Introducción. Gestión política, gestión administrativa y gestión histórica: ocultamientos y descubrimientos de los archivos de la represión", en: CATELA, Ludmila y JELIN, Elizabeth (compiladoras), Los archivos de la represión: documentos, memoria y verdad, Buenos Aires, Siglo XXI Editores, 2002. Pp. 1-13.

LARROBLA, Fabiana y FIGUEREDO, Magdalena. "De la "Ley de Caducidad" al "voto verde": nuevas formas de resistencia en marcos de legalidad. El Caso de la Comisión Nacional Pro Referéndum", Revista EUTOPÍAS, Biopolítica y memoria, Universidad de Valencia, España, Vol. 17, 2019, págs. 101-116. Disponible en: http://eu-topias.org/de-la-ley-decaducidad-al-voto-verde/

LARROBLA, Fabiana y LARROBLA, Carla. "Las investigaciones históricas de la Universidad de la República", Bajo la dirección de FERRARO, Maria H. y BOUVET, Lauriane, Memoria, Verdad y Justicia en Uruguay, ILCEA, Revista del Instituto de Lenguas y Culturas de Europa, América, África, Asia y Australia, 2016. Disponible en: https://journals.openedition.org/ilcea/3950

MARKARIAN, Vania y YAFFÉ, Jaime. Entrevista: “José Pedro Barrán: ¿Cómo pude haber escrito esto?”, en Revista Contemporánea: Historia y problemas del siglo XX, Volumen 1, Año 1, Montevideo, 2010. Pp. 179210.

MARKARIAN, Vania. "Los documentos del pasado reciente como materiales de archivo. Reflexiones desde el caso uruguayo", Revista Contemporánea: Historia y problemas del siglo XX, Año 7, Volumen 7, Montevideo, 2016. Pp. 177-191.

OBERTI, Alejandra, "Lo que queda de la violencia política. A propósito de archivos y testimonios" en Dossier Memorias de la Represión en América Latina, en Revista Temáticas, Revista de los Pos-Graduandos en Ciências Sociais, IFCH-Unicamp, Brasil, Año 17, número 34, 2009. Pp. 125-148. 
Presidencia de la República, Investigación Histórica sobre detenidosdesaparecidos en cumplimiento del artículo $4^{o}$ de la Ley de Caducidad, Tomo 1, Montevideo, IMPO, 2007.

RICO, Álvaro, ed. alt. Investigación Histórica sobre la dictadura y el Terrorismo de Estado en el Uruguay (1973-1985), 3 tomos, Montevideo, Udelar-CSIC, 2008.

RICO, Álvaro. "El actual malestar de los historiadores: entre la defensa del oficio y la responsabilidad política. Sobre archivo y repositorios documentos", Revista Contemporánea: Historia y problemas del siglo XX, Año 7, Volumen 7, Montevideo, 2016. Pp. 192-210.

RICO, Álvaro. Cómo nos domina la clase gobernante. Orden político y obediencia social en la democracia posdictadura. Uruguay 1985-2005, Montevideo, Trilce, 2005.

RICOEUR, Paul. La memoria, la historia, el olvido, Buenos Aires, Fondo de Cultura Económica, 2004.

SARLO, Beatriz. Tiempo pasado. Cultura de la memoria y giro subjetivo. Una discusión, Buenos Aires, Siglo XXI, 2005.

TODOROV, Tzvetan. Memoria del mal, tentación del ben. Indagación sobre el siglo XX, Barcelona, Ediciones Península, 2002.

RECEBIDO EM: 02/09/2019 APROVADO EM: 03/10/2019 